\title{
Étude ethnobotanique et screening phytochimique de Caesalpinia benthamiana (Baill.) Herend. et Zarucchi (Caesalpiniaceae)
}

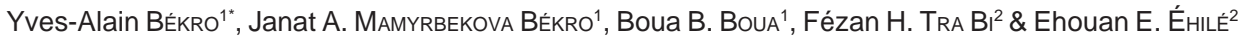 \\ ${ }^{1}$ Laboratoire de Chimie Bioorganique et de Substances Naturelles, UFR-SFA, Université d'Abobo-Adjamé, 02 BP 801 Abidjan 02 - \\ Côte d'Ivoire. \\ ${ }^{2}$ Laboratoire de Physiologie, Pharmacologie et de Phytothérapie, UFR-SN, Université d'Abobo-Adjamé, 02 BP 801 Abidjan 02 - Côte \\ d'Ivoire. \\ *Auteur pour les correspondances (E-mail : bekro2001@yahoo.fr) \\ Reçu le 02-08-2006, accepté le 12-02-2007.
}

\section{Résumé}

Les informations ethnobotaniques obtenues auprès de dix (10) tradithérapeutes du canton N'Gadié dans le Département d'Adzopé montrent que l'utilisation de Caesalpinia benthamiana (Caesalpiniaceae) ( $\mathrm{Cb}$ ) contribue significativement aux soins de santé primaire pour le traitement des troubles de l'érection. En effet, sur les quinze (15) espèces de plantes médicinales (réparties entre 14 familles) utilisées pour le traitement de diverses pathologies liées aux troubles de l'érection, $\mathrm{Cb}$ est la plus fréquemment prescrite dans ledit canton. Un screening phytochimique réalisé sur cinq (5) extraits bruts des racines de $\mathrm{Cb}$ a révélé qu'elles renferment des stérols, polyterpènes, flavonoïdes, saponines, tannins, sucres réducteurs, coumarines et des protéines.

Mots-clés: pharmacopée traditionnelle, Côte d'Ivoire, Adzopé, screening phytochimique, érection, Caesalpinia benthamiana (Caesalpiniaceae)

\begin{abstract}
Ethnobotanical study and phytochemical screening of Caesalpinia benthamiana (Baill.) Herend. and Zarucchi (Caesalpiniaceae)

Ethnobotanical Informations obtained beside ten (10) traditional herbalists of the canton N'Gadie in the Department of Adzope show that the use of Caesalpinia benthamiana (Caesalpiniaceae) (Cb) contributes significantly to primary health cares for the processing of erectile troubles. Indeed, on the fifteen (15) species of plant medicinal (distributed between 14 families) used for the various pathology processing linked to erectile troubles, $\mathrm{Cb}$ is the most frequently prescribed in the aforementioned canton. A phytochemical screening realized on five (5) extracted crude of roots of $\mathrm{Cb}$ has revealed that they contain the sterols, polyterpenes, flavonoids, saponines, tannins, reducing sugars, coumarins and proteins.
\end{abstract}

Key words: traditional pharmacopoeia, Côte d'Ivoire, Adzope, erection, Caesalpinia benthamiana (Caesalpiniaceae)

\section{Introduction}

Dans les pays africains, les plantes constituent par excellence, les principaux moyens médicamenteux pour des soins pratiques en santé publique. Kerharo et Adam (1950) rapportent qu'en médecine traditionnelle, certains organes de plantes, telles que les feuilles, les écorces, les racines sont utilisées dans le traitement d'affections courantes comme la 
bronchite, la trachéobronchite, etc., et ils trouvent aussi des applications en médecine dentaire. Les travaux de Diallo et al. (2001), indiquent que $80 \%$ de la population malienne ont recours à la médecine traditionnelle pour se soulager, voire traiter de nombreuses maladies. Au Togo et en Afrique du Sud, des plantes de la famille des Combretaceae trouvent usage en médecine traditionnelle pour leur activité antifongique (Batawila et al. 2005 ; Masoko et al. 2005). La pharmacopée égyptienne traditionnelle recommande fortement des extraits de plantes pour leur vertu anti-diarrhéique (Attia \& Samar, 2004). Au Sénégal, il a été montré que certaines plantes sont hypotensives. C'est le cas des graines de Parkia biglobosa qui manifestent une activité anti-hypertensive (Assane et al. 1993 ; Kouadio et al. 2000). En Côte d'Ivoire, Kouadio et al. (2000) démontrent par leur étude sur les activités analgésique et anti-inflammatoire de Parkia biglobosa que l'extrait de cette espèce est utilisé en tradithérapie. Selon Tra Bi (1997), le décocté de tiges de Paullinia pinnata et de feuilles de Lecaniodiscus cupanioides sert à traiter l'asthénie sexuelle. Tra Bi (1997) et Kouamé (1998) rapportent dans leurs travaux que Caesalpinia benthamiana aussi connue sous l'appellation Mezoneuron benthamianum, est utilisé dans le traitement de l'impuissance sexuelle. Ces exemples montrent bien que dans plusieurs pays du continent africain, les plantes ont toujours joué un rôle vital dans les soins médicaux, et que les moyens phytothérapeutiques sont riches, et continuent de s'enrichir en substances naturelles nouvelles. C'est pourquoi, il est important de faire une évaluation scientifique des plantes de notre pays afin d'en obtenir un maximum d'informations relatives à leur efficacité toxicologique, physiologique, pharmacodynamique, corrélée par les principes actifs qu'elles contiennent. De telles études permettraient de mettre au point des médicaments utilisables dans les soins de santé primaire à moindre coût.

Les troubles de l'érection sont un véritable problème de santé publique et aussi de société. L'acte sexuel est vital et son importance est loin d'être réduite à la fonction de reproduction. Les troubles de l'érection sont vécus péniblement car ils perturbent la qualité de vie des personnes qui en sont victimes ainsi que celle de leur partenaire. Selon les données épidémiologiques actuelles, 5 à 35\% des hommes en âge de procréer souffrent d'un dysfonctionnement érectile (Kubin et al. 2003). Cet état est souvent associé à certaines pathologies chroniques telles que le diabète, la neuropathie, la dépression etc. (Boua, 2005). Une étude réalisée par Opa (2003) entre 1998 et 2001 sur 104 patients, reçus en consultation au Centre Hospitalier de Cocody (Abidjan, Côte d'Ivoire), a révélé que $35,58 \%$ des patients souffraient de trouble de l'érection, $8,6 \%$ de trouble de l'éjaculation, $9,62 \%$ de trouble sexuel et $46,15 \%$ d'autres pathologies. Au nombre de ces patients, $56 \%$ ont présenté des défaillances psychologiques et $57,7 \%$ une impuissance de type psychogène. Une enquête menée en 1992 en Côte d'Ivoire sur un échantillon de 136 hommes diabétiques a montré que $44 \%$ d'entre eux ont présenté une impuissance sexuelle (Gomon, 2003 ; Boua, 2005). Face à ce fléau, des recherches sont en cours aux fins de trouver de nouvelles formes médicamenteuses efficaces. La pharmacopée et les médecines traditionnelles africaines peuvent y contribuer parfaitement en mettant au point des traitements qui présenteraient une efficacité palliative (Goldstein et al. 1998 ; Seagraves, 1998). Il existe en Côte d'Ivoire, un patrimoine floristique riche qui entre dans le traitement traditionnel des défaillances de l'érection. II s'agit entre autres de Securinga virosa (Euphorbiaceae), Parkia biglobosa (Menispermaceae), Turrea heterophylla (Meliaceae), Rauvolfia vomitoria (Apocynaceae), Waltheria indica (Euphorbiaceae), Caesalpinia benthamiana (Caesalpiniaceae) (Bokra, 2001 ; Boua, 2005). Différents organes de ces plantes sont utilisés, en médecine traditionnelle, soit sous forme de poudre à macérer dans l'eau ou l'alcool éthylique soit sous forme de cure-dent à mastiquer (Bokra, 2001). Selon des témoignages recueillis auprès des populations et des tradithérapeutes du canton N'Gadié dans le Département d'Adzopé (Sud Côte d'Ivoire) Caesalpinia benthamiana $(\mathrm{Cb})$ est la plus efficace parmi les plantes utilisées dans le traitement des troubles de l'érection (Boua, 2005). Or à notre connaissance, aucun travail relatif à la phytochimie de cette espèce n'existe dans la littérature. Dans le but de combler cette lacune, nous avons entrepris la présente étude relative à l'ethnobotanique et au screening phytochimique de $\mathrm{Cb}$. 


\section{Matériels et méthodes}

\subsection{Présentation de la zone d'étude}

Le canton N'gadié fait partie du Département d'Adzopé (Région de l'Agnéby), situé au Sud-est forestier de la Côte d'Ivoire. II est composé de cinq villages: Abousékakoi, Moapé, Ananguié, Ahouabo, Bouapé, et compte 2608 habitants sur 280340 du Département, soit $8,06 \%$ de cette population. Cette dernière est essentiellement composée des Attié, Agni parlant respectivement une langue d'origine nigéro-congolaise du groupe Kwa, sous-groupe Akan; des Sénoufo, peuple venu du Nord de la Côte d'Ivoire qui parle une langue nigéro-congolaise du groupe Gur (Anonymes, 1998, 2002) et d'autres peuples venus du Burkina-Faso, du Mali, du Ghana, et de la Mauritanie. Cette zone est caractérisée par un climat tropical humide (climat attiéen) et une végétation forestière dense et diversifiée.

\subsection{Méthode de travail pour l'étude ethnobotanique}

La méthode de travail a consisté à procéder par des enquêtes ethnobotaniques. Elles se sont déroulées en trois étapes dans le mois de mai 2006: l'entretien direct avec les populations et dix (10) tradithérapeutes de renom dont six (6) hommes et quatre (4) femmes (respectivement $60 \%$ et $40 \%$; moyenne d'âge 35 - 76 ans) sur les troubles de l'érection et les recettes recommandées pour le traitement; la sortie sur le terrain à la recherche des plantes utilisées dans les recettes et l'identification des différentes plantes récoltées.

\subsection{Informations ethnomédicales}

Les informations ont été collectées auprès des tradithérapeutes communément appelés «guérisseurs» de la zone d'étude. Parmi les plantes que nous avons recensées, et qui soignent plusieurs pathologies, $\mathrm{Cb}$ nous a été recommandée. Cette espèce a été identifiée et confirmée à l'herbier du Centre National Floristique (C. N. F.) de l'Université d'Abidjan Cocody, puis nommée selon la nomenclature de Hutchinson et Dalziel (1954) révisée par Jongkind (2004). Le nom de $\mathrm{Cb}$ en langue vernaculaire Attié est «Akpaba wun». Ses racines sont largement utilisées en usage médical traditionnel pour ses vertus aphrodisiaques. La posologie et le mode d'emploi traditionnels indiquent que la racine est utilisée une fois par jour au coucher sous forme de cure-dent.

\subsection{Matériel de criblage phytochimique}

Le matériel végétal est constitué de racines de $\mathrm{Cb}$. Elles ont été récoltées dans le mois de mai 2006 dans la forêt dense de Moapé. Ces organes ont été lavés sous un courant d'eau continu pendant $5 \mathrm{~min}$, découpés en petits morceaux et séchés à l'étuve à $50^{\circ} \mathrm{C}$ pendant 4 jours, puis pulvérisés avec un broyeur (marque RETSCH, type SM 100).

Les extraits bruts ont été obtenus par extractions successives avec des solvants en fonction de l'ordre croissant de leur polarité. Dans cet ordre, nous avons utilisé cinq solvants: hexane, chloroforme, acétate d'éthyle, méthanol, eau.

Deux cents (200) grammes de poudre végétale sont macérés dans 1 I d'hexane pendant $48 \mathrm{~h}$, puis filtrés sur Büchner. L'extrait brut hexanique (S1) obtenu est distillé sous vide jusqu'à siccité et on a obtenu $1,5 \mathrm{~g}(0,75 \%)$ de résidu (R1). Le marc est séché à la température ambiante puis macéré dans $1 \mathrm{I}$ de chloroforme pendant $48 \mathrm{~h}$. L'extrait brut chloroformique (S2) obtenu est distillé sous vide jusqu'à siccité pour donner 1,0 g $(0,5 \%)$ de résidu (R2). La même opération est reprise sur le marc séché avec respectivement 1 I d'acétate d 'éthyle, 1 | de méthanol et 1 | d'eau distillée portée à ébullition. Nous avons obtenu les extraits bruts d'acétate éthylique (S3), méthanolique (S4) et aqueux (S5) qui ont fourni $5,0 \mathrm{~g}(2,5 \%), 13,5 \mathrm{~g}(6,75 \%)$, et $9,0 \mathrm{~g}(4,5 \%)$ de résidus respectivement (R3), (R4) et (R5) après distillation sous vide (Koffi, 2003).

Pour les analyses par chromatographie sur couche mince (CCM), nous avons utilisé des chromatoplaques (gel de silice $60 \mathrm{~F}_{254}$ sur support aluminium) qui ont été révélées aux vapeurs d'iode $\left(I_{2}\right)$. Comme développants, les systèmes de solvants :

$\mathrm{C}_{6} \mathrm{H}_{6}-\mathrm{CH}_{3} \mathrm{COOEt} 30: 5$ et $\mathrm{CHCl}_{3}-\mathrm{MeOH}-\mathrm{H}_{2} \mathrm{O}$ $32: 17: 2,5$ ont été utilisés. Nous nous sommes servis d'un rotavapor (marque Büchi R110, type MKE 6540/2) pour les distillations sous vide.

\subsection{Criblage phytochimique}

Les tests de détection des grands groupes de 
composés chimiques ont porté sur les résidus (R1-R5). Nous nous sommes servis des techniques analytiques décrites dans les travaux de Lazurevskii et al. (1966) ; Senov (1978) ; Melenteva et Antonova (1993) ; Tona et al. (1998) et de Longanga et al. (2000).

\subsubsection{Détection des stérols et polyterpènes}

Les stérols et les polyterpènes ont été mis en évidence dans les résidus (R1 - R5) par la réaction de Liebermann. Un aliquote de résidu est dissoute à chaud dans $1 \mathrm{ml}$ d'anhydride acétique dans une capsule, puis reprise dans un tube à essai dans lequel sont coulés $0,5 \mathrm{ml}$ de $\mathrm{H}_{2} \mathrm{SO}_{4}$ concentré. L'apparition d'une coloration violette qui vire au bleu puis au vert indique une réaction positive.

\subsubsection{Détection des tannins}

Les tannoïdes (tannins hydrolysables) et les tannins vrais (tannins non hydrolysables ou condensés) sont des polymères de polyphénols. Ces derniers ont été mis en évidence par la réaction au chlorure de fer (III) dans les extraits bruts (S1 - S5).

À $2 \mathrm{ml}$ d'extrait brut sont additionnées quelques gouttes d'une solution aqueuse de $\mathrm{FeCl}_{3}$ à $2 \%$. L'apparition d'une coloration bleu-noir ou vert-noir indique respectivement la présence de tannoïdesou de tannins vrais.

Les tannins vrais ont été mis en évidence dans les résidus ( $R 3$ - $R 5$ ) par le réactif de Stiasny.

À un aliquote de résidu repris au $\mathrm{MeOH}$ puis évaporé, sont additionnés $15 \mathrm{ml}$ du réactif de Stiasny ( $30 \% \mathrm{CH}_{2} \mathrm{O}$ dans $\mathrm{HCl}$ concentré $2 / 1$ ). $\mathrm{La}$ formation de précipité sous forme de flocons après refroidissement, indique une réaction positive.

Les tannins vrais ont été également mis en évidence à chaud en présence de $\mathrm{HCl}$ concentré : à un aliquote de résidu repris dans $2 \mathrm{ml}$ d'eau distillée, sont ajoutées quelques gouttes de $\mathrm{HCl}$ concentré, le tout est chauffé au bain-marie bouillant. La formation d'un précipité rouge indique un test positif.

La mise en évidence des tannoïdes a été réalisée dans les résidus (R3 - R5) : aux filtrats des masses réactionnelles saturés à $\mathrm{CH}_{3} \mathrm{COONa}$, sont ajoutées quelques gouttes d'une solution aqueuse de $\mathrm{FeCl}_{3}$ à $2 \%$. La réaction est positive si une teinte bleu-noir apparaît.

\subsubsection{Détection des flavonoïdes}

Les flavonoïdes ont été mis en évidence dans les résidus ( $R 1$ - $R 5)$ par la réaction à la cyanidine. À un aliquote de résidu dissout dans $5 \mathrm{ml}$ d'éthanol chlorhydrique (2: $1, v / v)$ sont additionnés deux à trois copeaux de $\mathrm{Mg}$ (ou 30 $50 \mathrm{mg}$ de poudre de $\mathrm{Zn}$ ) et quelques gouttes d'isopentanol. L'apparition d'une coloration intense rose-orange ou violacée (rouge ou rouge-orange avec le $\mathrm{Zn}$ ) indique une réaction positive.

\subsubsection{Détection des quinones}

Les quinones ont été mises en évidence dans les résidus (R1 - R5) par le réactif de Borntraëger. Un aliquote de résidu dissout dans $5 \mathrm{ml}$ de $\mathrm{HCl}$ dilué au $1 / 5$, est chauffée au bain-marie bouillant pendant $30 \mathrm{~min}$, puis extraite avec $20 \mathrm{ml}$ de $\mathrm{CHCl}_{3}$ après refroidissement. À la phase organique, sont additionnés $0,5 \mathrm{ml}$ de $\mathrm{NH}_{4} \mathrm{OH}$ diluée à $50 \%$. L'apparition d'une teinte allant du rouge au violet indique une réaction positive.

\subsubsection{Détection des saponines}

Les saponines ont été mises en évidence dans les résidus (R1 - R5) par le test de mousse, puis leur présence confirmée par le test de sang et par la détermination de la densité optique (DO). Les résidus sont repris dans $5 \mathrm{ml}$ d'eau distillée, puis introduits dans un tube à essai. Le tube à essai est agité vigoureusement. La formation d'une mousse (hauteur supérieure à $1 \mathrm{~cm}$ ) stable, persistant pendant $1 \mathrm{~h}$ indique la présence abondante de saponines.

Le test de sang a été réalisé sur les extraits aqueux de R4 et R5. Dans un tube à essai contenant $2 \mathrm{ml}$ de sang frais d'animal dissout dans une solution physiologique (solution aqueuse de $\mathrm{NaCl}$ à $0,9 \%$ ), sont ajoutées quelques gouttes d'extrait aqueux. L'observation d'une décoloration par rapport à un tube témoin, indique un test positif.

Dans 3 tubes à essai, est introduit $1 \mathrm{ml}$ de solution sanguine ( $1 \mathrm{ml}$ de sang dans $25 \mathrm{ml}$ de solution isotonique). Un des tubes sert de témoin. Dans chacun des deux restants, sont ajoutées respectivement 5 et 10 gouttes d'extraits à tester. Après homogénéisation, le contenu des tubes à essai est centrifugé pendant $10 \mathrm{~min}$ à $2000 \mathrm{~g}$, puis, la DO de chaque surnageant 
prélevé est mesuré à l'aide d'un colorimètre (longueur d'onde $420 \mathrm{~nm}$ ).

\subsubsection{Détection des sucres réducteurs}

Les sucres réducteurs ont été mis en évidence dans les extraits bruts (S1 - S5) par le réactif de Fehling, puis, leur présence confirmée par le test de Tollens .

Pour réaliser le test de Fehling, à $5 \mathrm{ml}$ d'extrait brut sont additionnés $5 \mathrm{ml}$ de liqueur de Fehling. La formation d'un précipité rouge brique après 2-3 min de chauffage au bain-marie à $70^{\circ} \mathrm{C}$ indique une réaction positive.

La détection des sucres réducteurs par le test de Tollens a consisté à ajouter à $5 \mathrm{ml}$ d'extrait brut, $5 \mathrm{ml}$ du réactif de Tollens. La formation d'un miroir d'argent après quelques minutes indique une réaction positive.

\subsubsection{Détection des coumarines}

Les coumarines ont été mises en évidence dans les résidus (R1 - R5) par la réaction sur le cycle lactonique. Dans 2 tubes à essai, sont introduits $2 \mathrm{ml}$ de solution éthanolique obtenue à partir de chaque résidu. Dans un des tubes à essai, sont additionnés $0,5 \mathrm{ml}$ de $\mathrm{NaOH}$ à $10 \%$, puis les tubes à essai sont chauffés au bain-marie jusqu'à ébullition. Après refroidissement, sont rajoutés dans chaque tube à essai $4 \mathrm{ml}$ d'eau distillée. Si le liquide du tube à essai dans lequel l'on a ajouté la solution alcaline est transparente ou plus transparente par rapport au liquide du tube à essai témoin (sans solution alcaline), alors la réaction est positive. En acidifiant la solution transparente avec quelques gouttes de $\mathrm{HCl}$ concentré, elle perd sa coloration jaune, se trouble ou il se forme un précipité.

\subsubsection{Détection des protéines}

Les protéines ont été mises en évidence dans les résidus ( $\mathrm{R} 1-\mathrm{R} 5)$ par la réaction du biuret. À un aliquote de résidu dissout dans $2 \mathrm{ml}$ de $\mathrm{NaOH}$ aqueux à $20 \%$ dans un tube à essai, sont ajoutées 2 -3 gouttes d'une solution aqueuse de $\mathrm{CuSO}_{4}$ à $2 \%$. L'apparition d'une coloration violette, quelquefois avec une teinte rougeâtre, indique une réaction positive.

\subsubsection{Détection des alcaloïdes}

Les alcaloïdes ont été mis en évidence dans les résidus (R1 - R5) avec les réactifs de Dragendorff et de Burchard (réactifs de précipitation) : 0,1 g de résidu est repris dans $6 \mathrm{ml}$ d'éthanol à $60 \%$, puis reparti dans 2 tubes à essai. Dans le premier tube, sont ajoutées 2 gouttes de réactif de Dragendorff. L'apparition d'un précipité rouge orangé ou brun - rougeâtre indique un test positif. Dans le deuxième tube, sont ajoutées 2 gouttes de réactif de Burchard. L'apparition d'un précipité brun indique un test positif.

\section{Résultats et Discussion}

\section{1. Étude ethnobotanique}

L'entretien que nous avons eu avec les tradithérapeutes a permis de répertorier 15 espèces végétales réparties entre 15 genres et 14 familles comme l'indique le tableau 1. Ces plantes médicinales soignent diverses pathologies d'impuissance sexuelle. Caesalpinia benthamiana est la plante la plus utilisée (9 tradithérapeutes sur 10) parmi les plantes qui entrent dans les recettes du traitement de la dysfonction érectile. Cette indication thérapeutique de $\mathrm{Cb}$ et des autres espèces est aussi rapportée par d'autres peuples (Adjanohoun \& Aké Assi, 1979). Par ailleurs, les informations reçues auprès des tradithérapeutes nous relatent que les troubles de l'érection, bien que considérés comme un sujet tabou, est connu de tous dans le canton N'Gadié. Selon les tradithérapeutes interrogés, les causes de cette pathologie sont essentiellement organiques. Elles se manifestent par une affection génitale ou bien générale qui est l'hémorroïde, communément appelé $<<$ koko $>$. Cette maladie se présente sous deux formes, les troubles de l'érection et l'impuissance absolue. La première forme, plus critique, se caractérise par un pénis qui ne réagit à aucune stimulation. La deuxième forme se manifeste par des signes pathologiques : parfois l'érection est incomplète ; dans d'autres cas, elle débute normalement mais ne peut se maintenir suffisamment longtemps pour que l'acte sexuel soit normal ; la non persistance des érections matinales. C'est la dernière forme qui est la mieux traitée par les tradithérapeutes du canton N'Gadié (73,68\% des recettes totales). Cependant, eu égard à l'étude comparative entre la classification de Leclere (1990) et celle des tradithérapeutes, nous faisons les remarques suivantes : la première forme plus 
critique et la deuxième forme de cette maladie pourraient correspondre respectivement à l'impuissance absolue et aux troubles de l'érection. Les troubles de l'éjaculation comme par exemple l'éjaculation précoce, est considérée comme une forme d'impuissance sexuelle par Leclere (1990). En revanche, les tradithérapeutes la considère comme une forme de stérilité.

Tableau 1 : Répertoire des plantes utilisées contre les troubles de l'érection dans le Canton N'Gadié (Département d'Adzopé)

\begin{tabular}{|c|c|c|c|c|}
\hline Espèces de plantes & Noms locaux (Attié) & Organes utilisés & $\begin{array}{l}\text { Mode de } \\
\text { préparation }\end{array}$ & Mode d'administration \\
\hline $\begin{array}{l}\text { Carpolobia lutea } \\
\text { (Polygalaceae) }\end{array}$ & N'gbemi & Racine & Mastication & $\begin{array}{l}\text {-Voie orale } \\
-2 \text { fois par jour }\end{array}$ \\
\hline Clausena anisata (Rutaceae) & Agnimêtê & Racine & Mastication & $\begin{array}{l}\text {-Voie orale } \\
-1 \text { fois tous les } 2 \text { jours }\end{array}$ \\
\hline Diodia scandens (Rubiaceae) & Biebienan & Feuilles fraîches & Expression & $\begin{array}{l}- \text {-Voie orale } \\
-3 \text { à } 5 \text { gouttes dans } \\
\text { chaque narine au } \\
\text { coucher }\end{array}$ \\
\hline Garcinia kola (Clusiaceae) & Touakpê & & Mastication & $\begin{array}{l}\text {-Voie orale } \\
-2 \text { graines par jour }\end{array}$ \\
\hline Glyphaea brevis (Tiliaceae) & N'kobagbê & Feuilles séchées & $\begin{array}{l}\text { Macération (vin } \\
\text { de palme) }\end{array}$ & $\begin{array}{l}\text {-Voie orale } \\
\text { - toute la journée }\end{array}$ \\
\hline $\begin{array}{l}\text { Klainedoxa gabonensis } \\
\text { (Irvingiaceae) }\end{array}$ & Akpabeu & & Mastication & $\begin{array}{l}\text {-Voie orale } \\
\text { - } 5 \text { bourgeons matin et } \\
\text { soir }\end{array}$ \\
\hline $\begin{array}{l}\text { Caesalpinia benthamiana } \\
\text { (Caesalpiniaceae) }\end{array}$ & Akpaba wun & Racines fraîches & Mastication & $\begin{array}{l}\text {-Voie orale } \\
\text { - } 1 \text { racine par jour au } \\
\text { coucher }\end{array}$ \\
\hline $\begin{array}{l}\text { Microdesmis keayana } \\
\text { (Pandaceae) }\end{array}$ & Kpeuzun & $\begin{array}{l}\text { Feuilles fraîches + } \\
\text { amande de palme }\end{array}$ & Mastication & $\begin{array}{l}\text {-Voie orale } \\
-1 \text { fois tous les deux } \\
\text { jours }\end{array}$ \\
\hline $\begin{array}{l}\text { Ostryoderris leudocobotrya } \\
\text { (Fabaceae) }\end{array}$ & Prékèchi pékèssê & $\begin{array}{l}\text { Ecorce de tige }+ \\
\text { piment rouge }\end{array}$ & Mastication & $\begin{array}{l}\text {-Voie orale } \\
-1 \text { fois par jour }\end{array}$ \\
\hline \multirow{2}{*}{$\begin{array}{l}\text { Palisota hirsuta } \\
\text { (Commelinaceae) }\end{array}$} & \multirow{2}{*}{ Wouchiakpê } & $\begin{array}{l}\text { Feuilles fraîches + } \\
\text { piment }\end{array}$ & Pulpation & $\begin{array}{l}\text {-Voie orale } \\
-1 \text { verre matin et soir }\end{array}$ \\
\hline & & Moelle de la tige & $\begin{array}{l}\text { Macération } \\
\text { (eau) }\end{array}$ & $\begin{array}{l}\text {-Voie anale } \\
\text { - lavement } \\
\end{array}$ \\
\hline \multirow[t]{2}{*}{ Paullinia pinnata (Sapindaceae) } & \multirow[t]{2}{*}{ Tomgbi } & Ecorce de racine & Pulvérisation & $\begin{array}{l}\text {-Voie orale } \\
-1 \text { pincée dans une } \\
\text { bouillie ou dans une } \\
\text { sauce à raison d'une } \\
\text { fois par jour }\end{array}$ \\
\hline & & Tige & $\begin{array}{l}\text { Macération (vin } \\
\text { de palme) }\end{array}$ & $\begin{array}{l}\text {-Voie orale } \\
\text { - } 1 \text { verre par jour }\end{array}$ \\
\hline $\begin{array}{l}\text { Penianthus } \\
\text { zenkeri(Menispermaceae) }\end{array}$ & Zin zakoué & Racine de $5 \mathrm{~cm}$ & $\begin{array}{l}\text { Macération (vin } \\
\text { de palme) }\end{array}$ & $\begin{array}{l}\text {-Voie orale } \\
\text { - boire toute la journée }\end{array}$ \\
\hline Rinorea ilicifolia (Violaceae) & Nommon kpeur & $\begin{array}{l}\text { Tige, feuilles } \\
\text { fraîches }\end{array}$ & $\begin{array}{l}\text { Macération (vin } \\
\text { de palme) }\end{array}$ & $\begin{array}{l}\text {-Voie orale } \\
\text { - boire en une seule } \\
\text { prise }\end{array}$ \\
\hline $\begin{array}{l}\text { Sphenocentrum jollyanum } \\
\text { (Menispermaceae) }\end{array}$ & Hininzakoué & Racine fraîche & Mastication & $\begin{array}{l}\text {-Voie orale } \\
-1 \text { cure-dent par jour }\end{array}$ \\
\hline Waltheria indica (sterculiaceae) & Atosa & $\begin{array}{l}\text { Racine fraîche ou } \\
\text { sèche }\end{array}$ & $\begin{array}{l}\text { Décoction } \\
\text { (eau) }\end{array}$ & $\begin{array}{l}\text {-Voie orale } \\
-1 \text { verre matin et soir }\end{array}$ \\
\hline
\end{tabular}




\subsection{Criblage phytochimique}

Des 5 solvants utilisés pour les extractions, le méthanol est le solvant qui a produit la plus grande quantité de masse extraite $(6,75 \%)$, et l'hexane, la plus petite $(0,75 \%)$. Ces résultats suggèrent l'utilisation du méthanol comme solvant indiqué pour l'extraction de composés chimiques naturels.

Les résultats du tri phytochimique réalisé sur les extraits de racines de $\mathrm{Cb}$ sont mentionnés dans le tableau 2. Les quinones et les alcaloïdes sont absents de tous les extraits bruts. Les sucres réducteurs sont présents dans tous les extraits bruts, sauf dans l'extrait brut hexanique. Ces sucres réducteurs sont des monosaccharides et des disaccharides (Bruneton, 1999 ; Longanga et al. 2000). L'extrait brut hexanique (S1) contient des stérols, des polyterpènes et des coumarines. L'extrait brut chloroformique (S2) est composé de stérols, polyterpènes, coumarines, flavonoïdes et de sucres réducteurs. Ces résultats ont été confirmés par CCM (développant benzène - éthyle acétate $30: 5$ ) qui ont révélé une multitude de spots dans les extraits S1 et S2. Par ailleurs, selon Lazurevskii et al. (1966), le test de détection des coumarines met aussi en évidence les flavonoïdes et les quinones. Si ce test est positif en absence de flavonoïdes (test à la cyanidine négatif) et de quinones (test au réactif de Borntraëger négatif), comme c'est le cas dans notre étude, alors la détection de coumarines est confirmée. L'extrait brut acétate éthylique (S3) est composé de stérols, polyterpènes, flavonoïdes, sucres réducteurs, tannins et de protéines. Les extraits bruts méthanolique (S4) et aqueux (S5) renferment des stérols, polyterpènes, flavonoïdes, sucres réducteurs, tannins, protéines et des saponines. Nous avons confirmé ces résultats par $\mathrm{CCM}$ obtenue dans un développant plus polaire $\left(\mathrm{CH}_{3} \mathrm{Cl}\right.$ - $\left.\mathrm{MeOH}-\mathrm{H}_{2} \mathrm{O} 32: 17: 2,5\right)$, qui a présenté plusieurs spots conformément à nos attentes. En ce qui concerne les tannins, nous les avons détectés au moyen d'une solution aqueuse de $\mathrm{FeCl}_{3}$ à $2 \%$. L'apparition d'une coloration bleu-noir dénote de la présence de tannoïdes de la série de l'acide gallique. La présence des tannins vrais s'est confirmée par l'apparition d'une coloration vert-noir, qui ne peut être visible qu'en présence d'une couleur plus foncée (bleu-noir). À cet effet, nous avons réalisé le test à l'acide chlorhydrique concentré (spécifique aux tannins vrais) au terme duquel nous avons observé la formation d'un précipité rouge. Nous étions donc, en présence de tannins vrais de la série des catéchines. Les résultats ont affirmé aussi que S3, S4, S5 contenaient des tannins galliques et catéchiques. Ces résultats corroborent ceux obtenus similairement avec du thé vert par Lazurevskii et al. (1966). Les saponines sont abondamment présentes dans S4 et S5. Outre le test de mousse, leur présence a été mise en évidence par le test de sang, confirmé par la méthode spectrophotométrique.

Tableau 2 : Screening phytochimique des racines de Caesalpinia benthamiana (Caesalpiniaceae)

\begin{tabular}{|c|c|c|c|c|c|c|}
\hline \multirow{2}{*}{$\begin{array}{l}\text { Composés } \\
\text { phytochimiques }\end{array}$} & \multirow{2}{*}{$\begin{array}{l}\text { Réactions/ } \\
\text { Réactifs }\end{array}$} & \multicolumn{5}{|c|}{ Extraits bruts / Résidus } \\
\hline & & S1/R1 & S2/R2 & S3/R3 & S4/R5 & S5/R5 \\
\hline Stérols et polyterpènes & $\mathrm{RL}$ & ++ & + & ++ & ++ & + \\
\hline \multirow{2}{*}{ Tannins vrais } & $\mathrm{RS}$ & - & - & + & + & + \\
\hline & $\mathrm{HCl}$ & & & + & + & + \\
\hline \multirow{2}{*}{ Saponines } & TM & - & - & - & ++ & +++ \\
\hline & TS & & & & + & + \\
\hline \multirow{2}{*}{ Sucres réducteurs } & LF & - & ++ & ++ & +++ & + \\
\hline & $\mathrm{RT}$ & - & ++ & ++ & +++ & + \\
\hline Alcaloïdes & RB & - & - & - & - & - \\
\hline
\end{tabular}

$R L=$ Réaction de Liebermann ; RS = Réactif de Stiasny ; RC = Réaction à la Cyanidine ; RBG = Réactif de Borntraëger ; TM = Test de Mousse ; TS = Test de Sang ; LF = Liqueur de Fehling ; RT = Réactif de Tollens ; RSL = Réaction sur Lactones ; RB = Test du Biuret ; $R D=$ Réactif de Dragendorff $; R B=$ Réactif de Burchard $;(+)=$ présents $;(++)=$ abondants $;(+++)=$ très abondants ; $(-)=$ absents 
Les dosages spectrophotométriques ont montré que S5 contient plus de saponines. Ce groupe de composés est tensioactif (test de mousse positif) et possèdent des propriétés hémolytiques (test d'hémolyse positif). Ces propriétés sont liées à l'interaction des saponines avec les stérols de la membrane érythrocytaire ; ce qui entraîne une augmentation de la perméabilité de la membrane et un mouvement ionique : $\mathrm{Na}^{+}$et $\mathrm{H}_{2} \mathrm{O}$ entrent et $\mathrm{K}^{+}$ sort en favorisant l'éclatement de la membrane qui entraîne la fuite de l'hémoglobine. Aussi, note-t-on que les saponines manifestent une forte activité spermicide. C'est pour cette raison, que ce groupe de composés chimiques a été utilisé pour des essais de crèmes contraceptives destinées à l'application vaginale (Bruneton, 1999).

À notre avis, l'ensemble des résultats du screening phytochimique expliquerait en partie, et ce de façon rationnelle, l'engouement des tradithérapeutes en général et ceux du canton N'Gadié en particulier, à utiliser les plantes médicinales, notamment $\mathrm{Cb}$ aux fins de soins contre les troubles de l'érection. De nombreux exemples issus de travaux scientifiques tirés de la bibliographie étayent notre hypothèse. En effet, les écorces de Pausinystalia yohimbe sont riches en alcaloïdes indoliques dont les teneurs sont de 5-6\% (Paris \& Letouzey 1960) et $1-6 \%$ (Bruneton, 1999). Cette espèce est reconnue aphrodisiaque. Cette activité due à la vasodilatation marquée au niveau du bassin et du corps caverneux est liée à l'action de la yohimbine, l'alcaloïde majoritaire (60 à $70 \%$ ) (Pousset, 1992 ; Bruneton, 1999). Par ailleurs, les racines de Tabernanthe iboga, abrisseau de l'Afrique équatoriale, sont réputées aphrodisiaques. Cette activité biologique est liée à la présence d'alcaloïdes indoliques, ibogaïne (majoritaire), tabarnanthine, ibogaline, ibogamine que renferme l'écorce des racines (5 à 6\%) (Bruneton, 1999). Ces deux exemples démontrent clairement que certains alcaloïdes, notamment indoliques sont responsables des activités aphrodisiaques. Dans le cas de notre étude en revanche, le criblage phytochimique a prouvé que $\mathrm{Cb}$ ne contient pas d'alcaloïdes. À cet égard, nous pensons que son utilisation hautement recommandée dans le traitement des troubles érectiles serait probablement liée à la présence des différents groupes de composés chimiques que nous avons identifiés. Les résultats des études biologiques nous permettront de confirmer ou d'infirmer notre assertion.

\section{Conclusion}

L'étude ethnobotanique menée dans le canton N'Gadié dans le Département d'Adzopé a montré que $\mathrm{Cb}$ est la mieux indiquée pour le traitement de la dysfonction de l'érection parmi les 15 espèces végétales recensées. Le screening phytochimique réalisé sur les extraits de racines de $\mathrm{Cb}$ a prouvé par ailleurs, qu'elles contiennent différents groupes de composés chimiques qui seraient à l'origine de son activité biologique.

\section{Remerciements}

Nous remercions le professeur AKE-ASSI Laurent de I'Université d'Abobo-Adjamé pour sa contribution à l'identification des espèces.

\section{Références citées}

Adjanohoun E. \& Aké Assi. L., 1979. Contribution au recensement des plantes médicinales de Côte d'Ivoire. Ministère de la Recherche Scientifique, Centre National de Floristique (CNF). Abidjan, Côte d'Ivoire ; 358 pp.

Anonyme, 1998. Recensement démographique de Côte d'Ivoire. Abidjan, Côte d'Ivoire, Institut National de Géographie Tropical ; pp. 285.

Anonyme, 2002. Dictionnaire universel, AUF $4^{e}$ Edition Hachette, Paris (France); pp. 1508.

Assane M., Baba Moussa R., Bassene E. \& Sere A., 1993. Antihypertensive action of Parkia biglobosa (Kacq) Benth seeds in the rat. Dakar Med. 38 (1): 49-54.

Attia H. A. \& Samar M. M., 2004. Antidiarrhoeal activity of some egyptian medicinal plant extracts. J. Ethnopharmacol. 92 : 303-309.

Batawila K., Kokou K., Koumaglo K., Gbeassor M., De Foucault B., Bouchet Ph., \& Akpagana K., 2005. Antifungal activities of five Combretaceae used in Togolese traditional medicine. Fitoterapia 76: $264-268$

Bokra A. R., 2001. Études des effets physiologiques et pharmacodynamiques de quelques plantes utilisées dans la traitement de 
l'impuissance sexuelle masculine en Côte d'Ivoire. Thèse de doctorat en pharmacie, Université d'Abidjan. 205 pp.

Boua B. B., 2005. Études des effets pharmacodynamiques de Mezoneuron benthamianum (Caesalpiniaceae), une plante entrant dans le traitement de l'impuissance sexuelle. Mémoire de DEA, Université d'Abidjan Cocody. 59 pp.

Bruneton J., 1999. Parmacognosie - Phytochimie

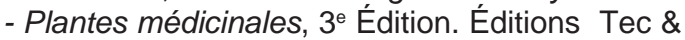
Doc et médicales internationales. F 94234 Cachan, Paris (France) ; 1120 pp.

Diallo D., Marston A., Terreaux C., Touré Y., Smestad Paulsen B. \& Hostettmann K., 2001. Screening of malian medicinal plants for antifungal, larvicidal, molluscicidal, antioxidant and radical scavenging activities. Phytother. Res. 15 : 401-406.

Goldstein I., Lue T., Padma-Nathan H., Roses R., Steers W. \& Wicker P., 1998. For the sildenofil study group. Oral sildenofil in the treatment of erectile dysfunction. New Engl. J. Med. 338: 1648-1659.

Gomon L., 2001. Impuissance sexuelle chez le diabétique en Côte d'Ivoire. Thèse de doctorat en médecine, Université d'Abidjan Cocody. 200 pp.

Hutchinson J. \& Dalziel J.M., 1954. Flora of West Tropical Africa. Second Édition, Millbank, London (England). $651 \mathrm{pp}$.

Kerharo J. \& Adam JG., 1950. La pharmacopée sénégalaise traditionnelle, plantes médicinales et toxiques. Vigot - Frères, Paris (France) ; pp. 579-581.

Koffi A., 2003. Valorisation de la pharmacopée africaine : étude toxicologique et pharmacologique de Ziziphus mauritania (Rhamnaceae), une plante réputée antihypertensive. Thèse de doctorat en pharmacie, Université d'Abidjan Cocody. 137 pp.

Kouadio F., Kanko C., Juge M., Grinaux N., Jean A., N'guessan Y.T. \& Petit J.Y., 2000. Analgesic and antiinflammatory activities of an extracts from Parkia biglobosa used in traditional medicine in the Ivory Coast. Phytother. Res. 14 : 635-637.

Kouamé N. F., 1998. Influence de l'exploitation forestière sur la végétation et la flore classées du Haut-Sassandra (Centre-ouest de la Côte d'Ivoire). Thèse de doctorat $3^{\mathrm{e}}$ cycle, Université d'Abidjan Cocody. 227 pp.
Kubin M., Wagner G. \& Fugl-Meyer A.R., 2003. Epidemiology of erectile dysfunction. Int. J. Impuis. Res. 15 : 63-71.

Leclere L., 1990. Généralités sur l'impuissance sexuelle masculine. Progrès en Urologie 20 : 450-599.

Longaga A. Otshudi, Vercruysse A. \& Foriers A., 2000. Contribution to the ethnobotanical, phytochemical and pharmacological studies of traditionally used medicinal plants in the treatment of dysentery and diarrhoea in Lomola area, Democratic Republic of Congo (RDC). J. Ethnopharmacol. 71: 411-423.

Lazureskii G.V., Terenteva I.V. \& Chamchurin A.A., 1966. Praticheskii raboti po khimii soedinienii. Édition Vischaya Chkola, Moscou (Russie); 334 pp.

Masoko P., Picard J. \& Eloff J.N., 2005. Antifungal activities of six South African Terminalia species (Combretaceae). J. Ethnopharmacol. 99: 301-308.

Melenteva G.A. \& Antonova L.A., 1993. Farmatsevticheskaya khimia. Édition Moskva Medicina, Moscou (Russie); 574 pp.

Opa A., 2003. Stratégies thérapeutiques actuelles dans la prise en charge médicale de l'impuissance sexuelle à propos de 104 patients reçus au Service d'Urologie du CHU de Cocody de 1998 à 2001. Thèse de pharmacie, Université d'Abidjan Cocody. 85 pp.

Paris R. \& Letouzey R., 1960. Répartition des alcaloïdes dans le Yohimbe. J. Agri. Trop. Bot . Appl. 7 : 256.

Pousset J.L., 1992. Plantes médicinales africaines. Possibilités de développement (Tom II). Agence de Coopération Culturelle et Technique. Ellipses, Paris (France); 159 pp.

Seagraves R.T., 1998. Editorial : pharmacological Era in the treatment of sexual disorders. J. Sex. marit. therap. 24: 67-68.

Senov P. L., 1978. Farmatsevticheskaya chimia. Édition Moskva Medicina, Moscou (Russie); 480 pp.

Tona L., Kambu K., Ngimbi N., Cimanga K. \& Vlietinck A. J., 1998. Antiamoebic and phytochemical screening of some Congolese medicinal plants. J. Ethnopharm. 61: 57-65.

Tra Bi F.H., 1997. Utilisation des plantes, par l'homme, dans les forêts classées du Haut-Sassandra et de Scio, en Côte d'Ivoire. Thèse de doctorat $3^{\mathrm{e}}$ cycle, Université d'Abidjan Cocody. 215 pp. 